\title{
Cerebellar strokes: a clinical outcome review of 79 cases
}

Zhi Xu Ng${ }^{1}$, MBBS, MRCSG, Wei Ren Eugene Yang${ }^{1}$, MRCSE, FRCS, Edwin Seet ${ }^{2}$, MBBS, MMed, Kiok Miang Koh ${ }^{1}$, MRCSE, FRCS,

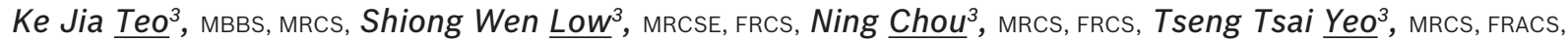

$N \underline{\text { Venketasubramanian }}^{4}$, MRCP, FRCP

INTRODUCTION Cerebellar infarcts and haemorrhages are relatively uncommon, accounting for less than $10 \%$ of all strokes. The objective of the present study was to quantify and compare the outcomes of patients with cerebellar infarct and those of patients with cerebellar haemorrhage, as well as to identify the risk factors that predict poor outcome in patients with cerebellar stroke.

METHODS We retrospectively reviewed the medical records of consecutive patients admitted to National University Hospital, Singapore, between 2004 and 2006, within one week of cerebellar stroke onset. Baseline data included demographics, concomitant comorbidities, and the presence or absence of brainstem compression and hydrocephalus (on computed tomography or magnetic resonance imaging). The Glasgow Outcome Scale and modified Rankin Score were used to assess outcome at discharge and at six months after discharge.

RESULTS A total of 79 patients with cerebellar stroke were admitted during the study period. Of these 79 patients, $17.7 \%$ died and $31.6 \%$ had poor outcomes at six months after discharge. Patients with cerebellar haemorrhage were found to be more likely to have poor outcomes as compared to patients with cerebellar infarct, both at discharge (odds ratio [OR] 4.3, 95\% confidence interval [CI] 1.3-14.1) and at six months after discharge (OR 5.2, 95\% Cl 1.6-17.2). When compared to small lesions $\left(<5 \mathrm{~cm}^{3}\right)$, lesions $>20 \mathrm{~cm}^{3}$ were significantly associated with poorer outcomes and the development of hydrocephalus and brainstem compression.

CONCLUSION Cerebellar strokes are a significant cause of morbidity and mortality. The outcomes of patients with cerebellar haemorrhage are more likely to be worse than those of patients with cerebellar infarct.

Keywords: cerebellum, haemorrhage, infarct, outcome

\section{INTRODUCTION}

Cerebrovascular disease is the third leading cause of death in the United States, accounting for $5.7 \%$ of total deaths in 2006. ${ }^{(1)}$ In Singapore, cerebrovascular disease is the fourth leading cause of death, accounting for $8.0 \%$ of deaths in $2009 .{ }^{(2)}$ Cerebellar strokes are relatively uncommon and account for less than $10 \%$ of all strokes. ${ }^{(3-5)}$ Due to tight space constraints, any space-occupying lesion in the posterior fossa may result in fourth ventricle and brainstem compression, leading to hydrocephalus and sudden death. ${ }^{(6-10)}$ Macdonell et al studied 2,000 consecutive stroke unit patients and showed that only $30(1.5 \%)$ patients had cerebellar infarction. ${ }^{(5)}$ Interestingly, the case fatality for cerebellar infarcts in the Macdonell study was $23 \%$, significantly higher than that for brain infarction at other locations. ${ }^{(5)}$ Da Pian et al also reported a high mortality rate of $38 \%$ for cerebellar haematomas. ${ }^{(11)}$

Controversy still exists regarding the most appropriate management for cerebellar strokes. ${ }^{(12-14)}$ Some neurosurgeons prefer conservative treatment, while others believe in early posterior fossa decompression. Although there are many published reports on cerebellar strokes, only a few have quantified the functional outcomes of cerebellar strokes. ${ }^{(5,7,8,11)}$ Therefore, the aim of the present study was to quantify and compare the differences in the outcomes of patients with cerebellar infarct and patients with cerebellar haemorrhage, as well as to identify risk factors that predict poor outcomes in patients with cerebellar strokes.

\section{METHODS}

Patients who were admitted to National University Hospital, Singapore, for cerebellar stroke between 1 January 2004 and 31 December 2006 were eligible for inclusion in this retrospective study. Approval for the study was granted by the institutional domain specific review board.

All of the patients included in the present study had undergone neuroimaging of the brain by computed tomography (CT) within one week of stroke onset, and had documented evidence of first-ever cerebellar infarct or cerebellar haemorrhage of hypertensive aetiology (Figs. 1 \& 2). Patients were excluded from the study if brainstem extension, synchronous supratentorial stroke or cerebellar haemorrhage of non-hypertensive aetiology (i.e. secondary to vascular malformation, warfarin usage or bleeding tendencies) was detected. Patients who had a history of non-cerebellar stroke with residual functional impairment (modified Rankin Scale [mRS] score $>1$ ) and patients with abnormal international normalised ratio secondary to anticoagulants, liver dysfunction or bone marrow disorders were also excluded. The demographic data (e.g. age, gender and comorbidities) of all the patients included in the present study was collected.

Calculation of the volume of infarct or haemorrhage was based on a formula derived from the ellipsoid method: volume $=$ (width $\times$ length $\times$ height $) \div 2$, where the maximum diameters of the infarct or haematoma were measured in three dimensions. ${ }^{(15)}$

${ }^{1}$ Division of Neurosurgery, Department of General Surgery, ${ }^{2}$ Department of Anaesthesia, Khoo Teck Puat Hospital, ${ }^{3}$ Department of Neurosurgery, ${ }^{4}$ Raffles Neuroscience Centre, Raffles Hospital, Singapore

Correspondence: Dr Ng Zhi Xu, Registrar, Division of Neurosurgery, Department of General Surgery, Khoo Teck Puat Hospital, 90 Yishun Central, Singapore 768828. kokoach2002@yahoo.com.sg 

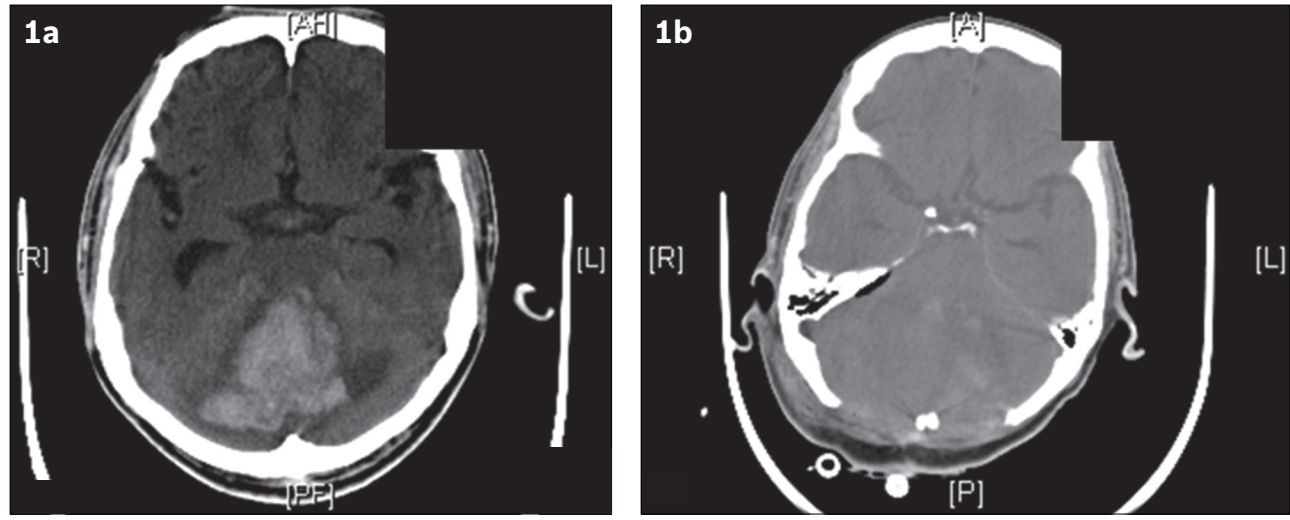

Fig. 1 (a) CT image at admission shows a large right cerebellar haemorrhage with effacement of the fourth ventricle, brainstem compression and resultant hydrocephalus. (b) CT image after surgical decompression (i.e. large posterior fossa craniectomy and evacuation of haematoma) shows improvement of the mass effect.
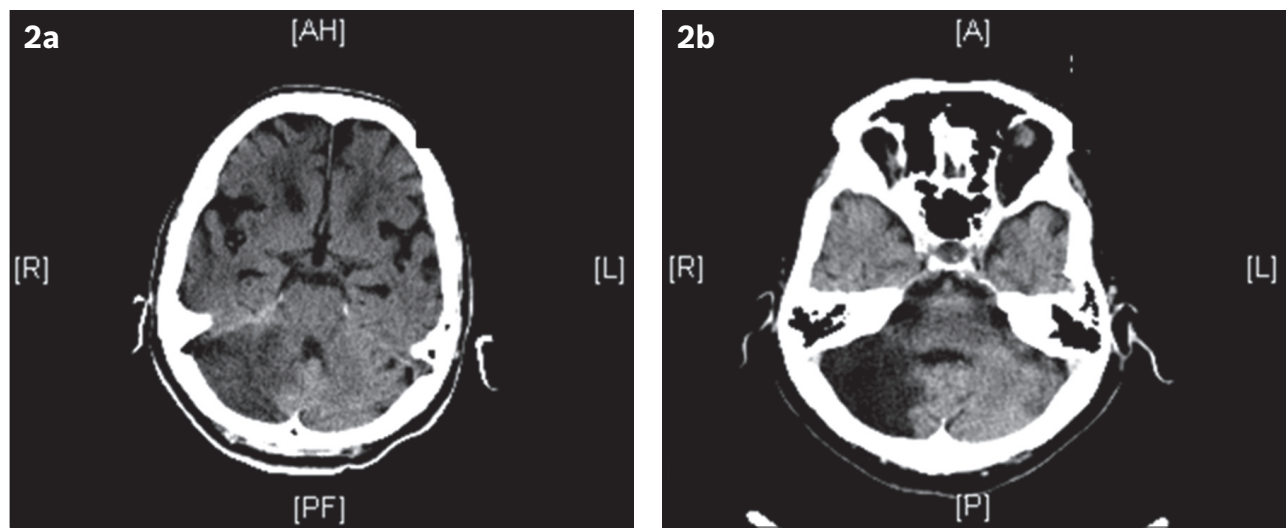

Fig. 2 (a) CT image at admission shows an acute right cerebellar infarct. The patient was admitted for severe giddiness and difficulty in walking. (b) CT image at follow-up six months after discharge shows a large area of encephalomalacia, consistent with a previous infarct.

The calculations were performed using the General Electric Centricity ${ }^{\circledR}$ program (GE Healthcare, Barrington, IL, USA), which was available in the hospital computers. The presence of brainstem compression or hydrocephalus was determined by a collaborator who was blinded to the clinical status of the patient.

All patients with cerebellar stroke were reviewed by a neurosurgeon within 24 hours of admission. Detailed neurologic examination was performed to determine the Glasgow Coma Scale on arrival (GCSOA), Glasgow Outcome Scale (GOS) and mRS scores of each patient. Patients with GCSOA score $>8$ were admitted to the neurosurgical high-dependency unit for observation. Patients with GCSOA score $\leq 8$ were intubated and managed in the surgical intensive care unit. Patients who showed deterioration of $>2$ points in the Glasgow Coma Scale (GCS) score underwent urgent repeat brain CT. If there was evidence of increasing mass effect or hydrocephalus secondary to cerebellar stroke, the patient was scheduled to undergo immediate surgery (within the hour). All patients with cerebellar infarct who did not require surgery were started on antiplatelet therapy.

The functional status of the patients was evaluated six months after discharge, in the neurosurgery outpatient clinic. The GOS and $\mathrm{mRS}$ scores at discharge and six months after discharge were obtained. ${ }^{(16,17)}$ Patients with GOS scores of 1,2 and 3, and patients with mRS scores of 3, 4, 5 and 6 were classified as having poor outcome.
All statistical analyses, including the comparison of outcomes between patients with cerebellar infarct and those with cerebellar haemorrhage (based on the GOS and mRS scores), and assessment of the differences in the outcomes of patients with and without hydrocephalus and brainstem compression, were performed using the Statistical Package for the Social Sciences version 17.0 (SPSS Inc, Chicago, IL, USA).

\section{RESULTS}

A total of 99 patients were admitted to our hospital for cerebellar stroke during the study period. However, only 79 of these patients fulfilled the criteria for inclusion. Three patients were lost to follow-up (at six months after discharge), leaving a total number of 76 patients for assessment of functional outcomes at six months after discharge. Of the 79 patients, 43 (54.4\%) patients were diagnosed with cerebellar infarction, while 36 (45.6\%) were diagnosed with cerebellar haemorrhage. Based on the TOAST classification, ${ }^{(18)} 30.1 \%$ of the cerebellar infarcts were due to atherosclerosis, $23.3 \%$ were due to cardioembolism, $6.9 \%$ were due to stroke of other determined aetiologies, and the remaining $39.7 \%$ were due to stroke of undetermined aetiology. The characteristics of the patients are shown in Table I.

Of the 79 patients, 20 underwent surgical treatment (Table II) 15 patients (4 infarcts, 11 haemorrhages) underwent urgent surgery based on the initial imaging findings and 5 patients ( 4 infarcts, 
Table I. Characteristics of the patients with cerebellar strokes $(n=79)$.

\begin{tabular}{|c|c|c|c|c|}
\hline \multirow[t]{2}{*}{ Characteristic } & \multicolumn{3}{|c|}{ No. of patients (\%) } & \multirow[t]{2}{*}{ p-value* } \\
\hline & Cerebellar infarct $(n=43)$ & Cerebellar haemorrhage $(n=36)$ & All $(n=79)$ & \\
\hline $\mathrm{Age}^{+, *}(\mathrm{yr})$ & $64.2 \pm 13.1$ & $67.2 \pm 11.5$ & $65.6 \pm 12.4$ & 0.68 \\
\hline Male patients & $27(62.8)$ & $19(52.8)$ & $46(58.2)$ & 0.37 \\
\hline GCSOA score s $^{\S}$ & $15.0 \pm 2.1$ & $14.0 \pm 4.9$ & $15.0 \pm 4.0$ & $<0.01$ \\
\hline Size of lesion ${ }^{t, 9}(\mathrm{~mL})$ & $16.9 \pm 16.5$ & $18.6 \pm 19.3$ & $17.7 \pm 17.8$ & 0.11 \\
\hline Location of cerebellar lesion & & & & 0.08 \\
\hline Purely hemispheric & $24(55.8)$ & $18(50.0)$ & $42(53.2)$ & \\
\hline Involvement of the vermis & $19(44.2)$ & $18(50.0)$ & $37(46.8)$ & \\
\hline Mortality & $4(9.3)$ & $10(27.8)$ & $14(17.7)$ & 0.03 \\
\hline \multicolumn{5}{|l|}{ Comorbidity } \\
\hline Hypertension & $30(69.8)$ & $29(80.6)$ & $59(74.7)$ & 0.05 \\
\hline Hyperlipidaemia & $23(53.5)$ & $3(8.3)$ & $26(32.9)$ & $<0.01$ \\
\hline Diabetes mellitus & $14(32.6)$ & $11(30.6)$ & $25(31.6)$ & 1.00 \\
\hline Ischaemic heart disease & $6(14.0)$ & $4(11.1)$ & $10(12.7)$ & 0.70 \\
\hline
\end{tabular}

$*_{p}$-value for mean age and size of lesion calculated using $t$-test with $95 \%$ confidence interval; $p$-value for male patients and mortality calculated using Chi-square test; $p$-value for GCSOA score calculated using Mann-Whitney $U$ test. $\dagger^{+D a t a}$ presented as mean \pm standard deviation. $¥$ The age ranges are as follows: cerebellar infarct 34-97 yr, cerebellar haemorrhage 41-96 yr, all patients 34-97 yr. ${ }^{\S}$ Data presented as median \pm standard deviation. 'Estimate based on computed tomography or magnetic resonance imaging. GCSOA: Glasgow coma scale on arrival

Table II. Treatment and mean size of lesion of patients admitted for cerebellar strokes $(n=79)$.

\begin{tabular}{|c|c|c|c|c|}
\hline Parameter & Cerebellar infarct $(n=43)$ & Cerebellar haemorrhage $(n=36)$ & All $(n=79)$ & p-value* \\
\hline \multicolumn{5}{|l|}{ Treatment $^{+}$} \\
\hline Conservative management & $35(81.4)$ & $24(66.7)$ & $59(74.7)$ & 0.32 \\
\hline Ventriculostomy & $1(2.3)$ & $2(5.6)$ & $3(3.8)$ & \\
\hline Suboccipital decompression & $7(16.3)$ & $10(27.8)$ & $17(21.5)$ & \\
\hline \multicolumn{5}{|l|}{ Size of lesion* $\left(\mathrm{cm}^{3}\right)$} \\
\hline Patients who underwent ventriculostomy & $9.0 \pm 0.0$ & $10.3 \pm 8.1$ & $9.9 \pm 5.7$ & 0.92 \\
\hline $\begin{array}{l}\text { Patients who underwent suboccipital } \\
\text { decompresssion }\end{array}$ & $34.5 \pm 23.2$ & $30.1 \pm 15.9$ & $31.9 \pm 18.7$ & 0.64 \\
\hline
\end{tabular}

*95\% confidence interval with unpaired student $t$-test. + Data presented as no. of patients $(\%)$. Data presented as mean \pm standard deviation.

1 haemorrhage) underwent surgery due to subsequent neurological deterioration in the hospital. Out of the 15 patients who underwent surgery based on initial imaging findings, 9 presented with poor GCSOA scores (median 3, range 3-8), while the remaining 6 patients, who had a median GCSOA scores of 14 (range 10-15), underwent surgery due to signs and symptoms of raised intracranial pressure. The mean time from admission to the development of neurologic deterioration was $17.5 \mathrm{hr}$.

Nine patients developed cerebrospinal fluid (CSF) leak from the postsurgical wound site. These nine patients were started on broad spectrum antibiotics for skin microorganisms to prevent CSF infection. None of the patients required the use of a lumbar drain for the treatment of CSF leak.

Among the patients in our study cohort, $38.0 \%$ of the patients had poor outcomes at discharge and $31.6 \%$ had poor outcomes at follow-up six months after discharge. When compared to the patients with cerebellar infarct, patients with cerebellar haemorrhage were more likely to have poor outcomes (based on GOS scores) at discharge (odds ratio [OR] 4.3, 95\% confidence ratio [Cl] 1.3-14.1, $\mathrm{p}=0.017$ ) and at follow-up six months after discharge (OR 5.2, $95 \% \mathrm{Cl} 1.6-17.2, \mathrm{p}=0.007$ ) (Table III). In our study cohort, 32.9\% of the patients developed hydrocephalus, while $26.6 \%$ developed compression of the brainstem. The presence of hydrocephalus (OR 6.3, 95\% Cl 1.5-26.9, $\mathrm{p}=0.014$ ) and compression of the brainstem (OR 3.1, 95\% Cl 0.6-15.4, p = 0.17) in patients with cerebellar stroke were also found to be associated with poorer outcomes based on GOS scores (Table IV). Three patients with hydrocephalus were eventually treated with ventriculoperitoneal shunt, while the rest were treated conservatively.

When patients with cerebellar strokes that were caused by lesions $<5 \mathrm{~cm}^{3}$ were compared with those who had cerebellar strokes that were caused by a lesion $>20 \mathrm{~cm}^{3}$, the latter group was found to be more likely to have poor outcomes (OR 9.6, 95\% Cl 2.5-36.8, $\mathrm{p}=0.001$ ), and develop hydrocephalus (OR 7.8, 95\% Cl 2.0-29.9, p = 0.003) and/or brainstem compression (OR 35.5, 95\% Cl 4.0-316.1, p = 0.001). There was no significant association between systolic blood pressure and site of lesion with the development of hydrocephalus $(p=0.39$ and $p=0.17$, respectively) and the development of brainstem compression ( $p=0.27$ and $p=0.43$, respectively).

A total of $14(17.7 \%)$ patients died $-4(5.1 \%)$ from the cerebellar infarct group and $10(12.7 \%)$ from the cerebellar 
Table III. Comparison of the outcomes of patients with cerebellar infarct and patients with cerebellar haemorrhage, at discharge and at follow-up six months after discharge.

\begin{tabular}{|c|c|c|c|c|c|c|}
\hline \multirow[t]{2}{*}{ Outcome } & \multicolumn{2}{|c|}{ No. of patients (\%) } & \multirow[t]{2}{*}{$\chi^{2}(\mathrm{df})$} & \multirow[t]{2}{*}{ p-value* } & \multirow[t]{2}{*}{ OR $(95 \% \mathrm{CI})$} & \multirow[t]{2}{*}{ p-value ${ }^{+}$} \\
\hline & Cerebellar infarct & Cerebellar haemorrhage & & & & \\
\hline \multicolumn{7}{|c|}{ Based on GOS score } \\
\hline At discharge & & & $6.15(1)$ & 0.013 & $4.3(1.3-14.1)$ & 0.017 \\
\hline Poor outcome & $11(25.6)$ & $19(52.8)$ & & & & \\
\hline Good outcome & $32(74.4)$ & $17(47.2)$ & & & & \\
\hline Poor outcome & $7(17.1)$ & $17(48.6)$ & & & & \\
\hline Good outcome & $34(82.9)$ & $18(51.4)$ & & & & \\
\hline \multicolumn{7}{|c|}{ Based on mRS score } \\
\hline At discharge & & & $7.06(1)$ & 0.008 & $4.4(1.3-15.3)$ & 0.019 \\
\hline Poor outcome & $27(62.8)$ & $32(88.9)$ & & & & \\
\hline Good outcome & $16(37.2)$ & $4(11.1)$ & & & & \\
\hline At follow-up $p^{\ddagger}$ & & & $11.9(1)$ & 0.001 & $5.2(1.8-14.4)$ & 0.002 \\
\hline Poor outcome & $13(31.6)$ & $25(71.4)$ & & & & \\
\hline Good outcome & $28(68.3)$ & $10(28.6)$ & & & & \\
\hline
\end{tabular}

*Confidence interval of $95 \%$ with Chi-square test. tOdds ratio of association was controlled for the presence of age, gender, size of lesion and comorbidities using logistic regression. $¥ 3$ patients ( 2 with cerebellar infarct and 1 with cerebellar haemorrhage) were lost to follow-up. Cl: confidence interval; df: degrees of freedom; GOS: Glasgow Outcome Scale; mRS: modified Rankin Scale; OR: odds ratio

Table IV. Comparison of outcomes at discharge (based on Glasgow Outcome Scale) of the patients with hydrocephalus and the patients with brainstem compression, secondary to cerebellar strokes.

\begin{tabular}{|c|c|c|c|c|c|c|}
\hline & \multicolumn{2}{|c|}{ No. of patients (\%) } & \multirow[t]{2}{*}{$\chi^{2}(\mathrm{df})$} & \multirow[t]{2}{*}{ p-value* } & \multirow[t]{2}{*}{ OR $(95 \% \mathrm{CI})^{+}$} & \multirow[t]{2}{*}{ p-value ${ }^{+}$} \\
\hline & Poor outcome $(n=30)$ & Good outcome (n = 49) & & & & \\
\hline Hydrocephalus & & & $20.3(1)$ & $<0.01$ & $6.3(1.5-26.9)$ & 0.014 \\
\hline Present $(n=26)$ & $19(73.1)$ & $7(26.9)$ & & & & \\
\hline Absent $(n=53)$ & $11(20.8)$ & $42(79.2)$ & & & & \\
\hline Brainstem compression & & & $22.4(1)$ & $<0.01$ & $3.1(0.6-15.4)$ & 0.17 \\
\hline Absent $(n=58)$ & $13(22.4)$ & $45(77.6)$ & & & & \\
\hline
\end{tabular}

* Confidence interval of $95 \%$ with Chi-square test. ${ }^{+}$Odds ratio of association was controlled for the presence of age and size of lesion and using logistic regression. $\mathrm{Cl}$ : confidence interval; OR: odds ratio

haemorrhage group. The average length of stay for the patients who had cerebellar infarct was 13 (range 2-56) days, while that of the patients with cerebellar haemorrhage was 12 (range $1-45)$ days.

\section{DISCUSSION}

Cerebellar strokes have been found to result in high morbidity and mortality. ${ }^{(11)}$ The results of the present study support this finding and provide evidence that patients with acute cerebellar strokes have a high likelihood of poor outcome and mortality. The outcomes were poorer for patients with cerebellar haemorrhage than for patients with cerebellar infarct. Patients who had cerebellar strokes that were associated with hydrocephalus or brainstem compression were also found to be more likely to have poorer outcomes. The reason for the difference in the outcomes of patients with cerebellar haemorrhage and those with cerebellar infarction is unclear. One possible explanation for the more sinister sequelae of cerebellar haemorrhage is that the continued bleeding after the start of a haemorrhage exerts a deadlier mass effect on the surrounding brain tissue and causes a more marked evolution of obstructive hydrocephalus (from the compression of the fourth ventricle and cerebral aqueduct). Although the analysis of the present study's results did not reveal any significant association between brainstem compression and poor outcomes, we believe that brainstem compression may still be clinically significant, as basic physiology describes the importance of the brainstem in the control of respiration, blood pressure, heart rate and cranial nerves. In other words, the involvement of the brainstem in stroke is likely to have significant outcomes.

In the present study, patients who had cerebellar strokes with lesions $>20 \mathrm{~cm}^{3}$ in volume were associated with worse outcomes and the development of hydrocephalus and brainstem compression. This knowledge will be useful for clinicians managing patients with cerebellar strokes. A lesion volume of $20 \mathrm{~cm}^{3}$ in cerebellar stroke patients can serve as a red flag, enabling proper counselling of patients and their relatives, in anticipation of a likely poor outcome and mortality risk. It can also be used to remind attending physicians to schedule a prompt neurosurgical consult.

In a previous study by St Louis et al, the authors showed that the following features on $\mathrm{CT}$ are strong predictors of poor 
outcome: (a) haematoma $>3 \mathrm{~cm}$ in diameter; (b) brainstem compression; (c) hydrocephalus; and (d) intraventricular extension from cerebellar haemorrhage. ${ }^{(19)}$ By applying the principles of the ellipsoid method, a cerebellar stroke with a lesion that is $3 \mathrm{~cm}$ in diameter on CT would give rise to a lesion with an estimated volume of $15 \mathrm{~cm}^{3}$. This is consistent with our finding that a cerebellar stroke lesion that has a volume $>20 \mathrm{~cm}^{3}$ is more likely to have a poor outcome.

In our study cohort, $25.3 \%$ of the patients underwent surgery, with similar distribution in both the cerebellar infarct and cerebellar haemorrhage groups. We found that patients who underwent suboccipital decompression fared significantly worse than those who were treated conservatively or who received ventriculostomy. This may be because patients with larger lesions on CT or poor GCSOA scores were more likely to require decompressive surgery as a definitive treatment. Since larger lesions were shown to be associated with poorer outcomes in the present study, the higher percentage of poor outcomes observed among the patients who underwent suboccipital decompression was not unexpected.

In a prospective study, Kirollos et al treated 50 consecutive patients using a protocol based on the appearance of the fourth ventricle and the GCS score. ${ }^{(20)}$ The mortality of the patients at three months was $40 \%$, with a sizeable proportion of patients dying from medical issues. None of the patients with a completely effaced fourth ventricle and coma (defined as GCS score $<8$ ) survived with a good outcome. The results of that study suggest that patients would fare better if they underwent surgery before clinical deterioration occurred. However, to date, the time point at which to start treatment, as well as the treatment of choice, remains controversial. Future studies evaluating whether surgery improves the outcomes of patients with cerebellar strokes are necessary. Based on the present study's results, it may be prudent to offer early surgical decompression, especially to patients with good GCSOA scores and stroke volumes $>20 \mathrm{~mL}$, as this may prevent the onset of brainstem compression, hydrocephalus and worse outcomes.

Retrospective studies are limited in that there is a lack of control over patients that are lost to follow-up. In the present study, we carefully vetted the records of all patients discharged from our department with a diagnosis of stroke and found that only 3 (3.8\%) patients were lost to follow-up. Another limitation of the present study was that the need and timing for surgical intervention was left to the discretion of the attending neurosurgeon. In other words, due to possible selection bias, there may be some difficulties in interpreting the outcome results. However, despite the retrospective nature of the present study and its relatively small sample size, we believe that the data obtained provides information that is useful to both clinicians and future research trialists.

To conclude, the results of the present study demonstrated that more than a third of patients with cerebellar stroke have poor outcomes such as death, vegetative state and severe disability.
Patients with cerebellar stroke lesions that have a volume $>20 \mathrm{~cm}^{3}$ were also found to be more likely to have worse outcomes and to develop complications such as hydrocephalus and brainstem compression.

\section{ACKNOWLEDGEMENTS}

We would like to thank Prof Ng Tze Pin, Department of Community, Occupational and Family Medicine, National University of Singapore, for his invaluable help and guidance on the calculation of statistics for the paper.

\section{REFERENCES}

1. Heron M, Hoyert DL, Murphy SL, et al. Deaths: Final Data for 2006. In: Centers for Disease Control and Prevention, National Vital Statistics Reports (2009) Volume 57, Number 14 [online]. Available at: http://www.cdc.gov/ nchs/data/nvsr/nvsr57/nvsr57_14.pdf. Accessed December 14, 2009.

2. Ministry of Health Singapore, Statistics, Principle Causes of Death. Available at: http://www.moh.gov.sg/mohcorp/statistics.aspx?id=5526. Accessed June 28, 2011.

3. Suzuki K, Kutsuzawa T, Takita K, et al. Clinico-epidemiologic study of stroke in Akita, Japan. Stroke 1987; 18:402-6.

4. Kim CH, Kim JS. Development of cerebral infarction shortly after intracerebral hemorrhage. Eur Neurol 2007; 57:145-9.

5. Macdonell RA, Kalnins RM, Donnan GA. Cerebellar infarction: natural history, prognosis, and pathology. Stroke 1987; 18:849-55.

6. St Louis EK, Wijdicks EF, Li H. Predicting neurologic deterioration in patients with cerebellar hematomas. Neurology 1998; 51:1364-9.

7. Sykora M, Diedler J, Jüttler E, et al. Intensive care management of acute stroke: surgical treatment. Int J Stroke 2010; 5:170-7.

8. Jensen MB, St Louis EK. Management of acute cerebellar stroke. Arch Neurol 2005; 62:537-44.

9. Cohen ZR, Ram Z, Knoller N, Peles E, Hadani M. Management and outcome of non-traumatic cerebellar haemorrhage. Cerebrovasc Dis 2002; 14:207-13.

10. Kase CS, Norrving B, Levine SR, et al. Cerebellar infarction. Clinical and anatomic observations in 66 cases. Stroke 1993; 24:76-83.

11. Da Pian R, Bazzan A, Pasqualin A. Surgical versus medical treatment of spontaneous posterior fossa haematomas: a cooperative study on 205 cases. Neurol Res 1984; 6:145-51.

12. Kobayashi S, Sato A, Kageyama Y, et al. Treatment of hypertensive cerebellar hemorrhage--surgical or conservative management? Neurosurgery 1994; 34:246-50.

13. Mathew P, Teasdale G, Bannan A, Oluoch-Olunya D. Neurosurgical management of cerebellar haematoma and infarct. J Neurol Neurosurg Psychiatry 1995; 59:287-92.

14. Salvati M, Cervoni L, Raco A, Delfini R. Spontaneous cerebellar hemorrhage: clinical remarks on 50 cases. Surg Neurol 2001; 55:156-61.

15. Stocchetti N, Croci M, Spagnoli D, et al. Mass volume measurement in severe head injury: accuracy and feasibility of two pragmatic methods. J Neurol Neurosurg Psychiatry 2000; 68:14-7.

16. Jennett $B$, Bond $M$. Assessment of outcome after severe brain damage. Lancet $1975 ; 1: 480-4$.

17. Wilson JT, Hareendran A, Grant M, et al. Improving the assessment of outcomes in stroke: use of a structured interview to assign grades on the modified Rankin Scale. Stroke 2002; 33:2243-6.

18. Adams HP Jr, Bendixen BH, Kappelle LJ, et al. Classification of subtype of acute ischemic strokes. Definitions for use in a multicenter clinical trial. TOAST. Trial of Org 10172 in Acute Stroke Treatment. Stroke 1993; 24:35-41.

19. St Louis EK, Wijdicks EF, Li H, Atkinson JD. Predictors of poor outcome in patients with a spontaneous cerebellar hematoma. Can J Neurol Sci 2000; 27:32-6.

20. Kirollos RW, Tyagi AK, Ross SA, van Hille PT, Marks PV. Management of spontaneous cerebellar hematomas: a prospective treatment protocol. Neurosurgery 2001; 49:1378-86. 